\title{
Fatigue crack growth analysis on square prismatic with embedded cracks under tension loading
}

\author{
M.S. Shaari ${ }^{1 *}$, A.K. Ariffin' ${ }^{1}$, Akiyuki Takahashi ${ }^{2}$, S. Abdullah ${ }^{1}$, Masanori Kikuchi² \\ and M.R.M. Akramin ${ }^{3}$
}

${ }^{1}$ Department of Mechanical and Materials Engineering, Faculty of Engineering \& Built

Environment, Universiti Kebangsaan Malaysia, 43600 Bangi, Malaysia

*Email: mohdshamil.shaari@yahoo.com

Phone: +60389216136

${ }^{2}$ Tokyo University of Science, 2641 Yamazaki, Noda, Chiba 287-8510, Japan

${ }^{3}$ Universiti Malaysia Pahang, 26600 Pekan, Pahang, Malaysia.

\begin{abstract}
One of the most important issues yet to be overcome by engineers is the integrity and reliability of engineering structures. This is to ensure the safety of the engineering structure is at the greatest since the catastrophic failures usually occur due to fatigue crack growth. Due to insufficient studies on the fatigue embedded crack growth, the prismatic bar is chosen as the model of the structure. It is wise to select the solid bar since the analysis can be much simpler, thus making it easier to examine the behaviour of the fatigue crack growth. In this study, the metallic square prismatic with embedded cracks is analysed using S-version Finite Element Modelling (S-version FEM) under tension loading. The S-version FEM is an open source program, that is built from codes previously compiled as a program. The S-version FEM structured using the global-local overlay technique consists of two separate global and local meshes. By using the basic concept from the energy release rate and stress intensity factors (SIF), the behaviour of the fatigue crack growth is analysed. From the linear elastic fracture mechanics concept, the SIF is calculated using the virtual crack closure-integral method. The influences of different initial crack size and aspect ratios on the fatigue crack growth are investigated in this study. In addition, the SIF results from the S-version FEM are compared with the analytical solutions. From the analysis, the root mean square errors (RMSE) are performed to support the validation. The RMSE shows a very small error of $0.227,0.086$ and 0.3089 according to the aspect ratio of $0.5,1.0$ and 2.0, respectively. The results also show significant characteristics and behaviour of the SIF trend along the crack front, corresponding to different aspect ratios. From this study, the S-version FEM is suitable to be used to predict the fatigue crack growth for the cracks embedded in a structure. Subsequently, the S-version FEM is an open source program can be modified for increasingly complex engineering problems.
\end{abstract}

Keywords: Fatigue analysis, fatigue crack growth rate, 3-D embedded cracks, S-version FEM.

\section{INTRODUCTION}

For more than four decades, many researchers have worked on the fatigue and fracture on various engineering structures. However, the deterioration of the engineering 
structures still becomes a challenge for the engineers to overcome. Several examples of engineering structures are used often in automotive components, aircraft manufacturers, bridges, buildings and many more [1]. This deterioration phenomenon occurs due to the cyclic stress, known as fatigue and should be detected before it reaches the threshold. The threshold occurs at stresses below the monotonic yield strength and for many years, researchers have conducted various tests to predict the fatigue due to the relationship with the safety of human beings [2-7]. Therefore, it is considered as one of the critical area for researchers to study in order to prevent any catastrophic incidents. One of the most critical engineering structures is the nuclear power plant. It not only benefits consumers by providing them with electricity, but it also reduces the level of pollution tremendously compared to plants operated using coal and gas. However, every structure will have the tendency to deteriorate. When that happens, the nuclear power plant will become the worst enemy for the consumers due to the radiation leakage [3]. Therefore, researchers were then and currently working on studies to prevent the nuclear power plant structure from deteriorating. Non-destructive testing (NDT) is commonly used to perform the structural integrity for a nuclear power plant and oil and gas structures [8]. In addition, a study related with numerical computation especially on the crack growth is still insufficient due to most crack growth studies only being related to surface cracks and not internal cracks $[9,10]$. The cracks are not only initiated from the surface of a structure, but could also start from the inside thereof [11-13]. Thus, it is very crucial to configure the behaviour of the embedded crack since it is not visible to the naked eye.

The numerical computation is used in various engineering fields, especially when it relates to the structural analysis for the oil \& gas pipeline, vehicle components or nuclear power plant $[14,15]$. For the past two decades, researchers have started to appreciate the use of numerical computations in solving numerous engineering problem [16]. It is commonly used to investigate the power plant [15], in automotives or aircraft $[17,18]$ and for the fatigue life assessment [12, 13, 19-23]. Nowadays, there are several commercial software applications that are available in the market for finite element modelling (FEM) [24-26]. These software applications are not only expensive, but only several of them are able to simulate crack propagation appropriately. In addition to the FEM, there are techniques that were developed to enhance the existing FEM. The Sversion of the finite element method (S-version FEM) is one of the techniques developed to improve the computational process and its results. The original concept of the $S$-version FEM was developed by Fish [27] and the author has highlighted the word "S" for superimposed meshes. The simplest explanation regarding the superimposed mesh is that global and local meshes are both overlaid to focus on the local effects, such as holes or cracks, and to reduce the computational effort especially on large structures. In this study, the embedded crack is located inside the prismatic bar and the finite element analysis is performed using the S-version FEM. The embedded crack is referred to as local mesh and the prismatic bar is referred to as global mesh. The biggest contribution by using the superimposed technique is the computation process only focusing on the local domain instead of on the whole domain used by the existing FEM software. A special subroutine is needed to embed the cracks inside the structure while using the superimposed technique. The S-version FEM is developed from codes before being compiled as an open source program. The concept and formulation using the S-version FEM technique will be discussed in the next section.

In order to simulate the crack propagation, the SIF needs to be calculated. In general, the calculation of the SIF is calculated by applying the virtual crack closureintegral method (VCCM) [28]. The theory of linear elastic fracture mechanics entails the 
benefit of computing the energy release rate on the nodal forces and displacements. It is known that both of them are the results of the finite element method. Several researchers also confirm that by calculating the SIF, it is essential to indicate the distribution of the crack growth along the crack front [25, 29]. In this study, the SIF for the embedded cracks are being compared with the analytical solutions by Newman \& Raju [30] corresponding to the difference in aspect ratios. The work presented in this study is a continuous study performed for surface cracks [9]. It can be used for various engineering problems, such as multiple embedded cracks, multi-axial fatigue, heat affected zones and more since it is an open-source program. In this study, the S-version FEM is used to predict the fatigue crack growth on a square prismatic bar with embedded cracks under tension loading.

\section{METHODS AND MATERIALS}

\section{Concept and Formulation of the S-Version FEM}

The concept of the S-version FEM is shown in Figure 1. In this study, the S-version FEM is used to simulate a single embedded crack under tension loading. A much simpler definition of the S-version FEM is the superposition mesh or the superimposed mesh. It consists of a global (coarser mesh) and local mesh (finer mesh) and both are superimposed in order to reduce the computation time without jeopardising the accuracy. The global mesh, $\Omega^{G}$, is generated according to the geometry before getting overlaid by the local mesh, $\Omega^{L}$. The local mesh is representing the shapes and sizes of the cracks, correspondingly. The boundary of each region is represented by $\Gamma$, where the superimposed boundary is represented by $\Gamma^{G L}$, whilst the boundary of the displacement and force (traction) is represented by $\Gamma^{u}$ and $\Gamma^{t}$ respectively.

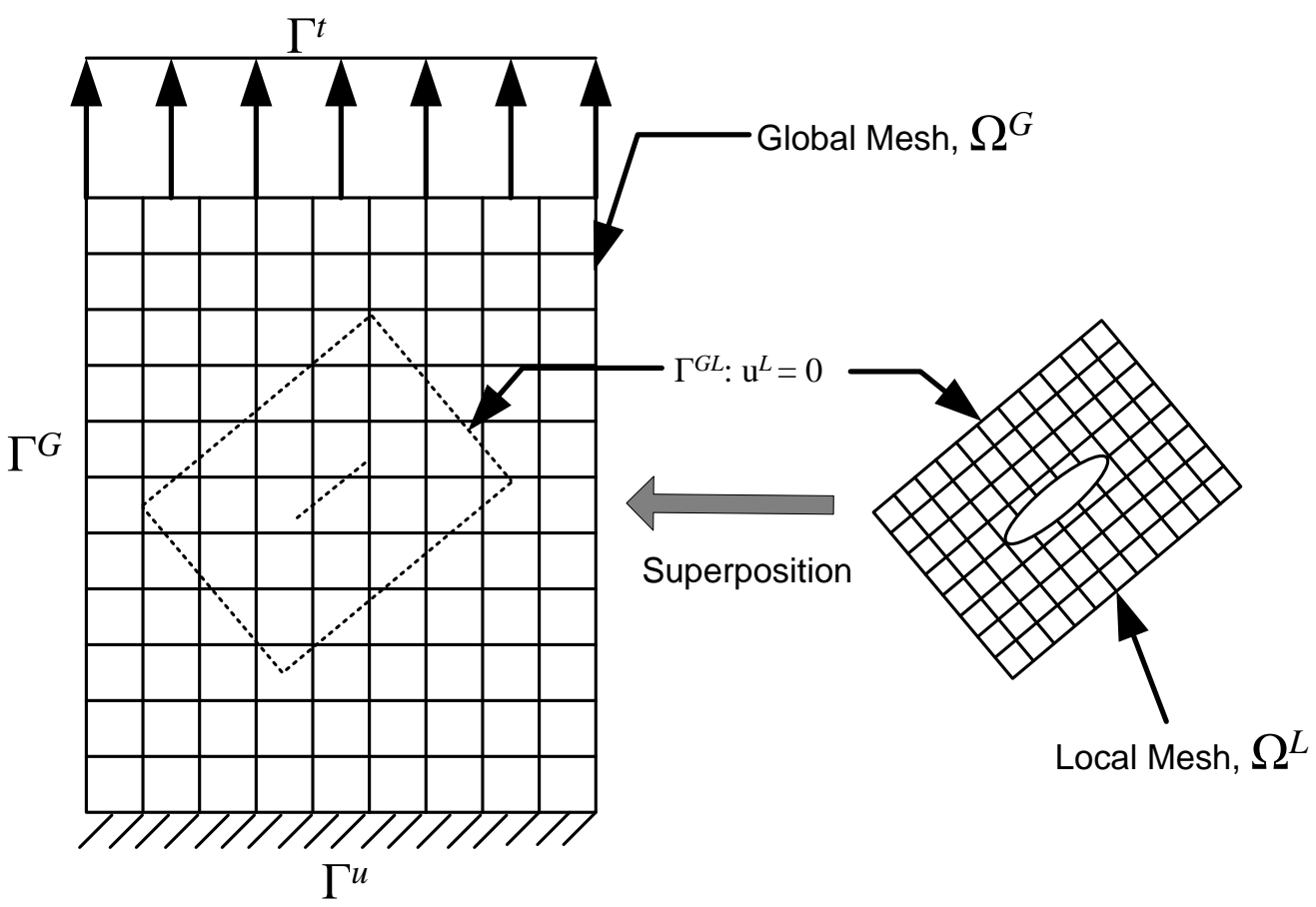

Figure 1. Concept of the S-version FEM.

The displacement function is defined independently for each global and local area. The displacement, $u$, for each is represented by $u^{G}$ and $u^{L}$ for the global area and local area, respectively. As shown in Figure 1, the displacement for the local area, $u^{L}$, is 
assumed to be zero to ensure the continuity of the global area and local area boundary. Respectively, Eq. (1) indicates the displacement function for the global and local area whilst Eq. (2) refers to the virtual work principle;

$$
u_{i}=\left\{\begin{array}{cccc}
u_{i}{ }^{G} & i & \in \Omega^{G}-\Omega^{L} \\
u_{i}{ }^{L}+u_{i}{ }^{L} & i & \in \Omega^{L} \\
u_{i}{ }^{L}=0 & i & \in \Gamma^{G L}
\end{array}\right.
$$

From the virtual work principle:

$$
\int_{\Omega} \delta u_{i, j} D_{i j k l} u_{k, l} d \Omega-\int_{\Omega} \delta u_{i, j} b_{i} d \Omega-\int_{\Gamma^{t}} \delta u_{i} t_{i} d \Gamma
$$

The displacement functions in Eq. (1) are then applied to the virtual work principle in Eq. (2) and shown in Eq. (3):

$$
\begin{aligned}
& \int_{\Omega^{G}} \delta u_{i, j}^{G} D_{i j k l} u_{k, l}^{G} d \Omega+\int_{\Omega^{L}} \delta u_{i, j}^{G} D_{i j k l} u_{k, l}^{L} d \Omega \\
& +\quad \int_{\Omega^{L}} \delta u_{i, j}^{L} D_{i j k l} u_{k, l}^{G} d \Omega+\int_{\Omega^{L}} \delta u_{i, j}^{L} D_{i j k l} u_{k, l}^{L} d \Omega \\
& =\int_{\Gamma^{t}} \delta u_{i}^{G} t_{i} d \Gamma^{t G}
\end{aligned}
$$

Thus, the final form of the S-version FEM is obtained in this following matrix form, as shown in Eq. (4):

$$
\left[\begin{array}{ll}
K_{G G} & K_{G L} \\
K_{L G} & K_{L L}
\end{array}\right]\left\{\begin{array}{l}
u^{G} \\
u^{L}
\end{array}\right\}=\left\{\begin{array}{l}
F_{G} \\
F_{L}
\end{array}\right\}
$$

where, the final form of the matrix is obtained and shown in Eq. (5)

$$
\begin{aligned}
{\left[K_{G G}\right] } & =\int_{\Omega^{G}}\left[B^{G}\right]^{T}[D]\left[B^{G}\right] d \Omega^{G} \\
{\left[K_{G L}\right] } & =\int_{\Omega^{L}}\left[B^{G}\right]^{T}[D]\left[B^{L}\right] d \Omega^{L} \\
{\left[K_{L G}\right] } & =\int_{\Omega^{L}}\left[B^{L}\right]^{T}[D]\left[B^{G}\right] d \Omega^{L} \\
{\left[K_{L L}\right] } & =\int_{\Omega^{L}}\left[B^{L}\right]^{T}[D]\left[B^{L}\right] d \Omega^{L} \\
\left\{F_{G}\right\} & =\int_{\Omega^{G}} N^{G} b_{i} d \Omega+\int_{\Gamma^{G}} N^{G} f_{i} d \Gamma \\
\left\{F_{L}\right\} & =\int_{\Omega^{G}} N^{L} b_{i} d \Omega+\int_{\Gamma^{G}} N^{L} f_{i} d \Gamma
\end{aligned}
$$

Notice, the $\left[K_{G L}\right]$ and $\left[K_{L G}\right]$ matrix is symmetric and it represents the stiffness matrix of the superimposed area. $[B]$ is the element strain-displacement matrix and matrix $[D]$ represents the stress-strain relationship of the material [31]. The nodal forces for the global and local areas are the $\left\{F_{G}\right\}$ and $\left\{F_{L}\right\}$, respectively. By using the final matrix of the S-version FEM in Eq. 4, the displacement can be calculated for each node simultaneously for both global and local cases. As the local mesh is growing accordingly, the global mesh is not changing along since the only region of interest is within the 
boundary of the crack front. The re-meshing process did also not include the global mesh; hence, the use of the superposition technique can reduce the computation time. The stress intensity factor (SIF) is calculated along with the expansion of the local mesh by using the energy release rate formulation.

The SIF is mainly used to predict the crack growth by computing the stress state at the crack tip. In order to obtain the SIF, the energy release rate must be first calculated by using several established methods. The virtual crack closure method (VCCM) is one approach that computes the energy release rate. The VCCM was developed by Rybicki [32] in 1977. The energy release rate, $G$, formula is shown by the following equation:

$$
G=\frac{1}{2 h}\left\{\left(u_{\alpha}^{\text {Upper }}+u_{\alpha}^{\text {Lower }}\right)\left(f_{\alpha}\right)+\left(u_{\beta}^{\text {Upper }}+u_{\beta}^{\text {Lower }}\right)\left(f_{\beta}\right)\right. \text {, }
$$

where

$$
\begin{aligned}
& u=u^{G}+u^{L} \\
d u= & \left(u_{G}+u_{L}^{\text {Upper }}\right)-\left(u_{G}+u_{L}^{\text {Lower }}\right) \\
= & \left(u_{L}^{\text {Upper }}+u_{L}^{\text {Lower }}\right)
\end{aligned}
$$

Eq. (7) is important to acquire the changes in the displacement by using the VCCM. Where the $\alpha$ and $\beta$ are both representing the nodal points for the forces, $f$, and displacement, $u$, near the crack tip. Figure 2 shows details of the illustration for the VCCM and the meaning of the upper and lower nodal forces and displacement.

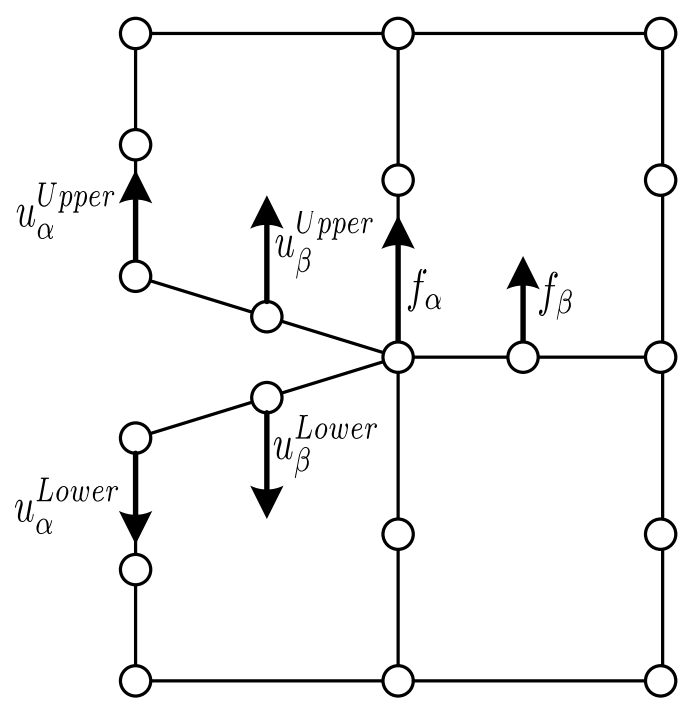

Figure 2. Concept of VCCM.

Once the energy release rate is obtained, the SIF can be computed by using the following equation;

$$
K_{I}=\sqrt{E G_{I}}, \quad K_{I I}=\sqrt{E G_{I I}}, \quad K_{I I I}=\sqrt{2 \mu l}
$$

where $K_{I}, K_{I I}$ and $K_{I I}$ are the mode I, mode II and mode III types of loading. The most widely known Paris' law [33] is very essential to predict the fatigue crack growth rate. It is suitable to be used for the $2 \mathrm{D}$ or $3 \mathrm{D}$ problem and the following equation shows Paris' law: 


$$
\frac{d a}{d N}=C\left(\Delta K_{e q}\right)^{n}
$$

where, $d a / \mathrm{dN}$ is the crack growth rate and $N$ is the number of cycles. The Paris' law constant is selected according to the material chosen, correspondingly $C$ is $1.67 \times 10^{-12}$ and $n$ is 3.23 , both emanates from the material constants.

The consideration of the crack growth rate and crack growth direction is needed, especially for mixed-mode loading cases. In this study, reference is only made to pure mode I loading conditions, but the crack growth direction and equivalent stress intensity factors, $\Delta K_{e q}$ are still taken into account and represented correspondingly by Eq. (10) and Eq. (11). Therefore, the S-version FEM is able to compute the mixed-mode type of loading, by integrating the Richard criterion into the codes. Hence, the equivalent stress intensity factors equation is used in this study. Richard et al. have always been focusing on the study of crack growth and developed a criterion for the crack growth direction. The details of explanations can be found from $[34,35]$.

$$
\varphi_{0}=\mp\left[140^{\circ} \frac{\left|K_{I I}\right|}{K_{I}+\left|K_{I I}\right|+\left|K_{I I I}\right|}-70\left(\frac{\left|K_{I I}\right|}{K_{I}+\left|K_{I I}\right|+\left|K_{I I I}\right|}\right)^{2}\right.
$$

where $\varphi_{0}<0^{\circ}$ for $K_{I I}>0$, and $\varphi_{0}>0^{\circ}$. Notice that the $K_{I I}$ value is affecting the direction of the crack, $\varphi_{0}$ as shown in

Figure 3. It shows the crack growth angle used to calculate the direction for the crack propagation along the crack front. The equivalent SIF, $\Delta K_{e q}$ is based on Richard's criterion expressed by Eq. (11).

$$
\Delta K_{e q}=\frac{\Delta K_{I}}{2}+\frac{1}{2} \sqrt{\Delta K_{I}^{2}+4\left(1.155 \Delta K_{I I}\right)^{2}+4\left(\Delta K_{I I I}\right)^{2}}
$$

where $\Delta K_{I}, \Delta K_{I I}$, and $\Delta K_{I I I}$ represent the range of stress intensity factor for mode I, mode II and mode III, respectively.

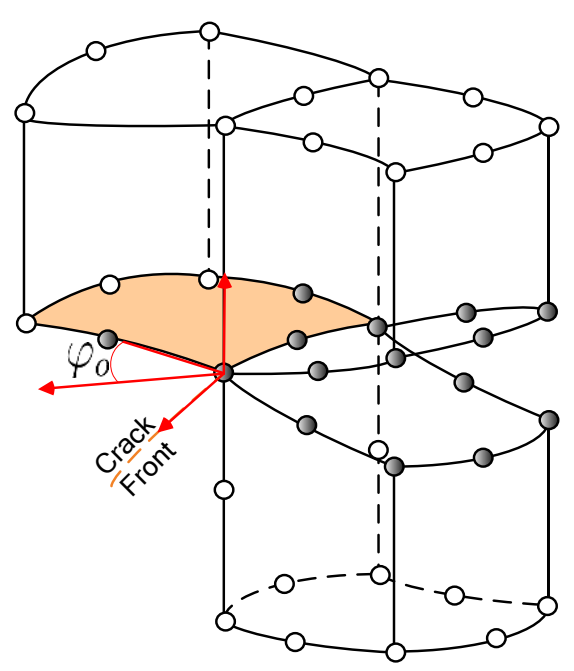

Figure 3. Crack growth angle. 


\section{RESULTS AND DISCUSSION}

\section{D Embedded Crack Simulation}

This section will be presenting the $3 \mathrm{D}$ embedded crack simulation. As mentioned earlier in this paper, the S-version FEM is an open source program. It is built in codes and written in $\mathrm{C \#}$ programming language and compiled into an open source program. All the formulations discussed in the last section were used to develop this program and the results will be presented and discussed in this section. The S-version FEM is coded and compiled using the Intel C\# Compilers (ICC). The program is divided into three, the pre and post-processor and also the engine (formulation of the S-version FEM). The preprocessor will be used to model the structure, determining the boundary conditions and material properties. Figure 4 is showing a simpler illustration for the location and the boundary condition of the simulation. The initial crack is located at the middle of the square prism and is perpendicular to the loading axis. The geometry of the square prismatic is $20 \mathrm{~mm}$ by $20 \mathrm{~mm}$ with a length of $80 \mathrm{~mm}$. Note that in this paper, $a$ is representing the crack depth and $c$ is the crack length. Boundary conditions were set to repeat the tension test. The bottom of the plate is fixed in all directions and the upper side is pulled with a uniform load of $100 \mathrm{MPa}$. The material selected for the square prism is A533B steel. The steel is a high toughness material and it is desirable for evaluating the energy release rate [36]. The material properties of the steel alloy consist of $210 \mathrm{GPa}$ for the modulus Young's $(E)$ and 0.29 for the Poisson ratio $(v)$.

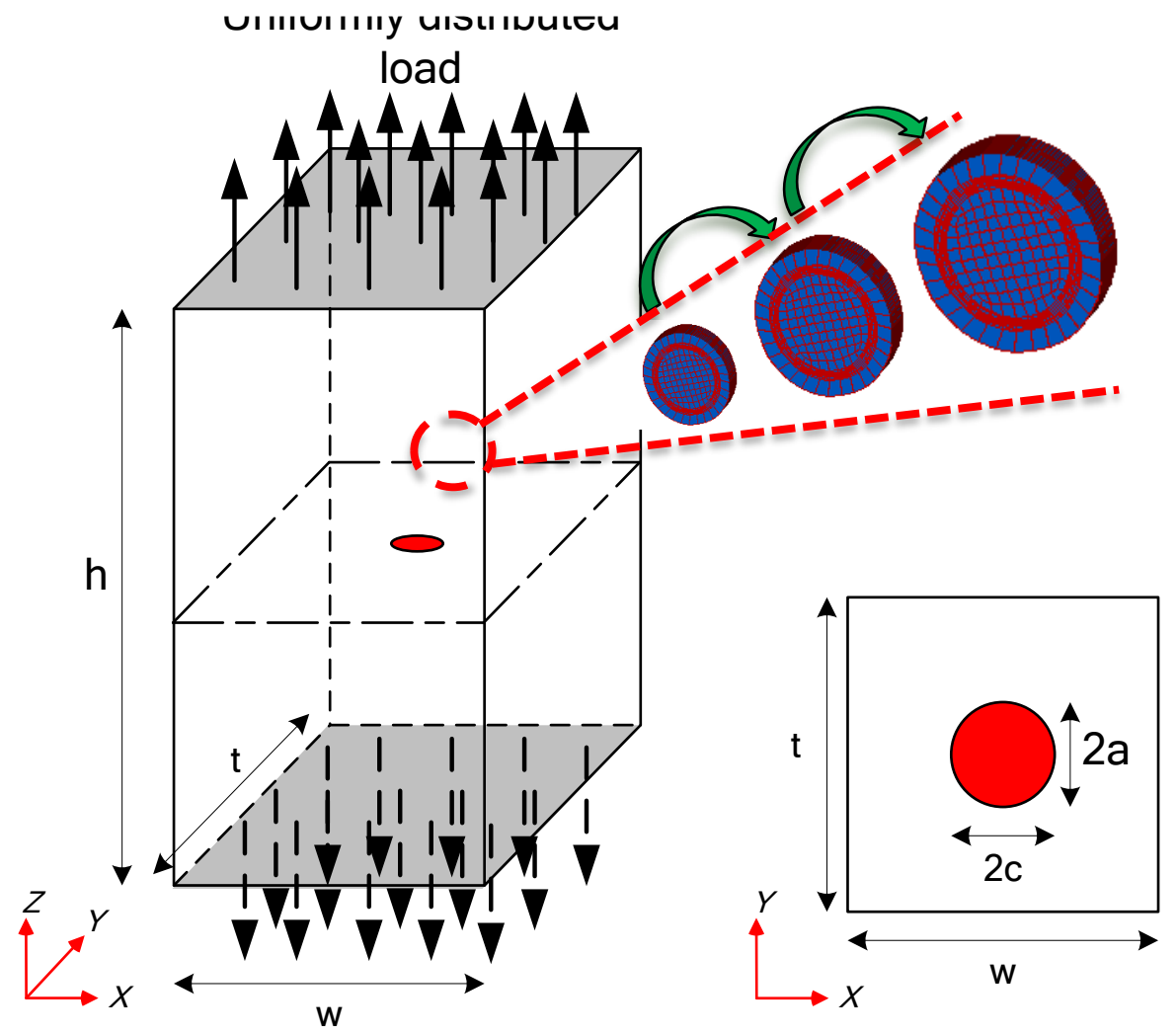

Figure 4. Location of the embedded crack and simulation conditions. 
The original concept of the S-version FEM was developed by Fish [27] following the linear elastic fracture mechanics (LEFM) concept. The author has highlighted the word superimposed meshes in the research paper. The simplest explanation regarding the superimposed mesh is that the global and local meshes are both overlaid. It is focusing on the local effect such as void or cracks with effort in reducing the computational effort especially on large structures. The figure below is showing the pre-processor of the $\mathrm{S}$ version FEM. The post-processor results will be presented later on in the next section. The structure is modelled and hexahedral meshes are used in this simulation. Figure 5(a) illustrates the square prismatic bar also known as the global mesh.

Figure 5(b) indicates the embedded crack further known as the local mesh. The local and global meshes were modelled using the 20-node hexahedral element. The hexahedral element is used to enhance the capability of the S-version FEM to calculate the VCCM [37].

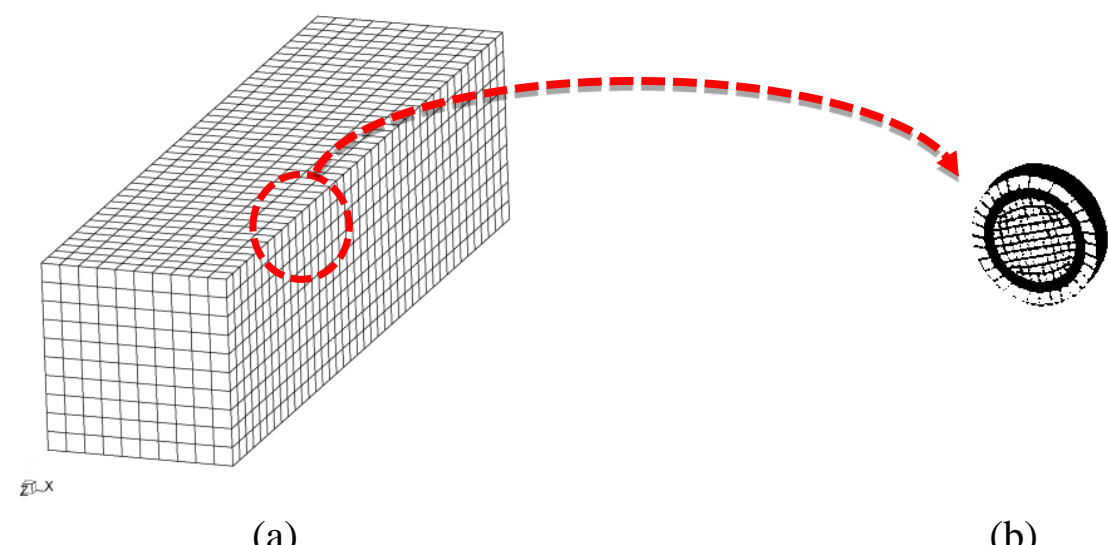

(a)

(b)

Figure 5. Pre-processor of the S-version FEM (a) global mesh of a square prismatic bar.

(b) Local mesh of the embedded crack.

\section{Crack Growth Behaviour Corresponding to the Aspect Ratios}

This section will be discussing and presenting the result of fatigue embedded crack growth corresponding to three different aspect ratios. The behaviour of the crack growth due to the aspect ratio to characterise the behaviour of the SIF trend and the how the fatigue embedded crack propagated. The crack growth behaviour corresponding to different aspect ratios is constructed from the results of the stress intensity factors (SIF). To differentiate the sizes and shapes of the aspect ratios, the local crack's length varies from $2 \mathrm{~mm}$ to $4 \mathrm{~mm}$ to resemble the aspect ratios as shown in

Table 1. The selected case study in this paper is using an aspect ratio $(a / c)$ of $0.5,1.0$ and 2.0 respectively, besides using a constant stress ratio $(R)$ of 0.1 . The $R=0.1$ is often used for tension-tension cyclic loading, where $\sigma_{\min }=0.1 \sigma_{\max } . \sigma_{\min }$ is the minimum stress and $\sigma_{\max }$ is the maximum stress applied to the prismatic bar, relatively from the cyclic loading history. Furthermore, $R=0.1$ is used by several researchers for the fatigue testing of an oil \& gas pipeline [10,25], aircraft components and nuclear power plants [29].Several researchers used the 0.1 stress ratio to study the crack propagation for semielliptical surface cracks $[9,10]$, the crack propagation for plasticity-induced crack closures [38] and multiple crack interactions [39]. $a$ is the crack depth and $c$ is the crack 
length. Figure 6 shows the differences in crack shapes between the three aspect ratios. The 1.0 aspect ratio is in a perfect circular shape and it will be used as a benchmark for the 0.5 and 2.0 aspect ratio. Hypothetically, the SIF trend along the crack front using 1.0 $a / c$ is the most stable without a significant fluctuation. The reason will be discussed later on in this paper. Figure 7 is giving a better illustration to understand the position and angular direction for the crack front. This is important in order to subsequently understand the SIF results. The results for the embedded fatigue crack growth will be presented and discussed. Three different shapes and sizes of the embedded crack are located inside a square prismatic. Each of the cracks is consecutively simulated using the S-version FEM and aspect ratios $(a / c)$ of $0.5,1.0$ and 2.0.

Figure 8 is presenting the SIF results for all three aspect ratios compared with the analytical solution from Newman \& Raju [30]. The comparison shows a very good agreement between the results obtained by FEM and closed form solution.

Table 1. Initial crack sizes and aspect ratios.

\begin{tabular}{lll}
\hline $\begin{array}{l}\text { Crack Length, } c \\
(\mathrm{~mm})\end{array}$ & $\begin{array}{l}\text { Aspect } \\
(a / c)\end{array}$ & $\begin{array}{l}\text { Stress } \\
\text { Ratio, } R\end{array}$ \\
\hline 4 & 0.5 & 0.1 \\
2 & 1 & 0.1 \\
2 & 2 & 0.1 \\
\hline
\end{tabular}

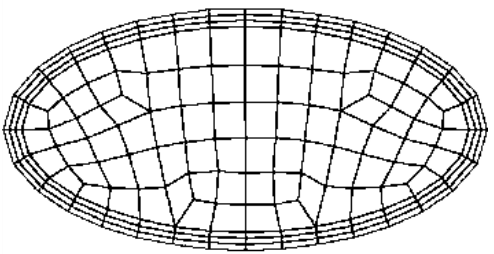

(a)

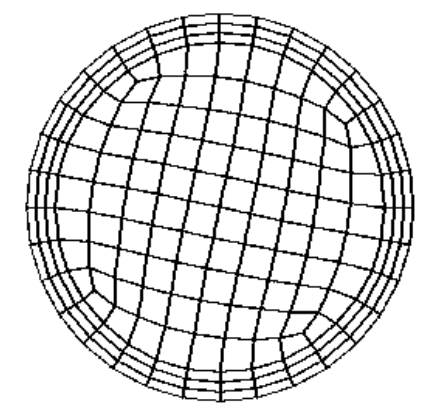

(b)

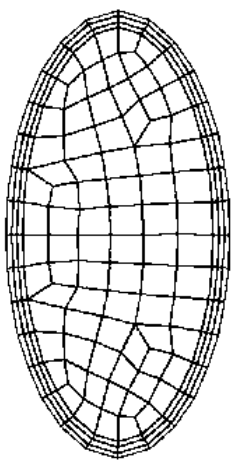

(c)

Figure 6 Embedded crack corresponding to the aspect ratio $(a / c)$, (a) 0.5 , (b) 1.0, (c) 2.0 


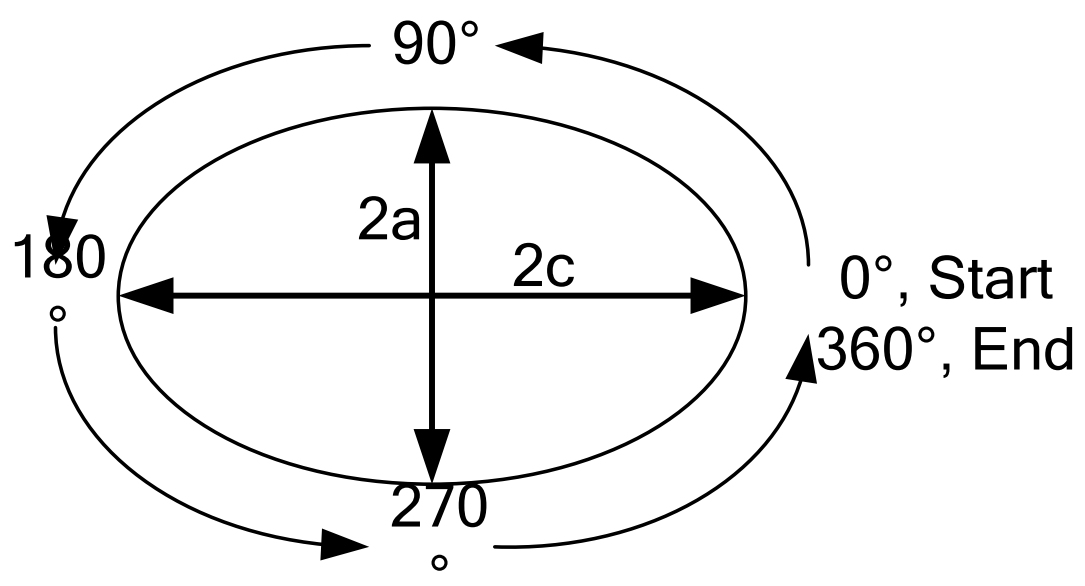

Figure 7. Position and angular direction of the crack front.

From the graph, it is clear that the $a / c$ of 1.0 shows almost a flat trend along the crack front, similar to the hypothesis mentioned in the last paragraph. The angular direction $(\theta)$ is referred in order to determine the SIF value along the crack front. This is due to the SIF value indicating the possibility for the crack to be propagated. Hence, wherever the SIF's value is large, there is a higher tendency for the crack to grow. The highest SIF is recorded from the 2.0 aspect ratio. This happens because the value of SIF does indeed reflect onto the crack types, shapes and sizes. The reason is because of the fatigue crack growth occurring at the largest area of the crack.

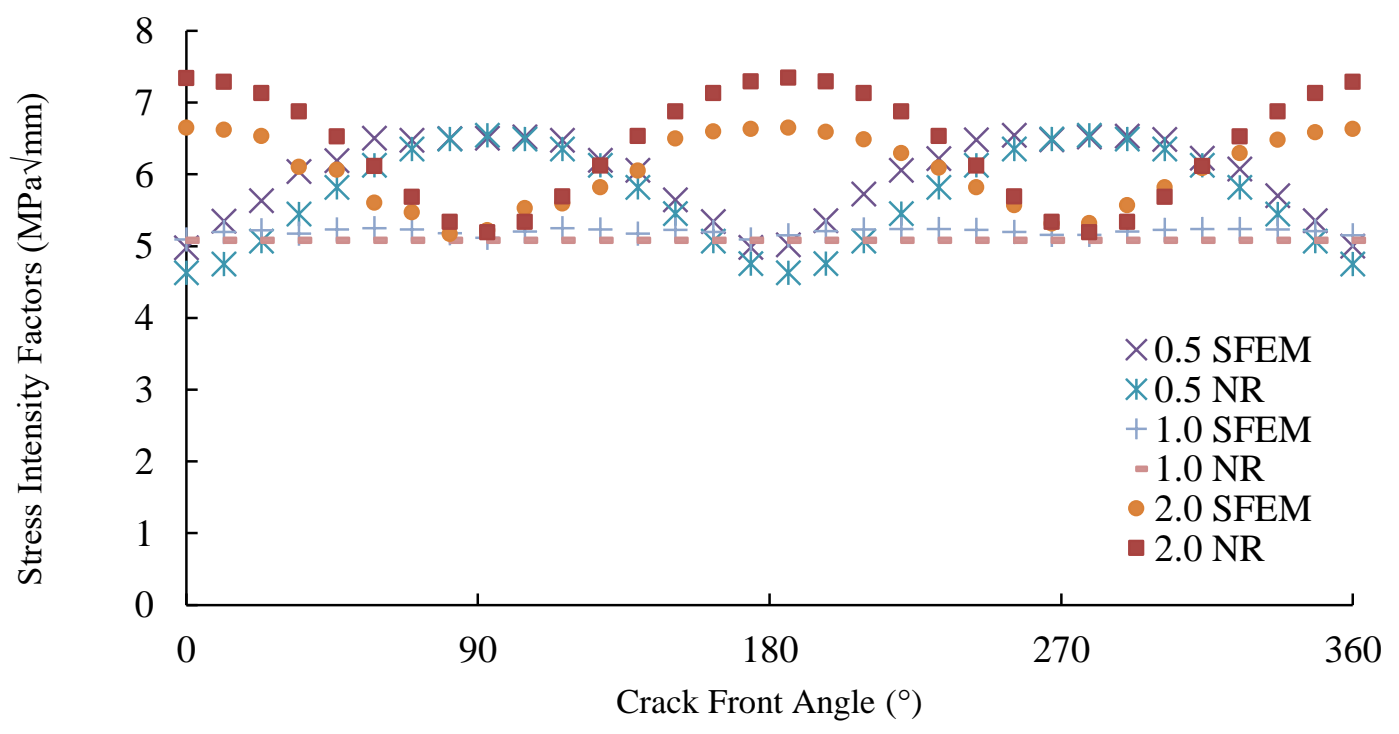

Figure 8. Comparison of stress intensity factors, KI between the S-version FEM and Newman and Raju's [30] for a 0.5, 1.0 and 2.0 aspect ratio $(a / c)$.

That is the reason why the highest value of the SIF is recorded at the location where the crack can easily propagate, instead at the tight and corner area. In addition, the correlation analysis is performed to ensure the accuracy of the results. The RMSE results show a low value of error between both the S-version FEM and Newman \& Raju [30]. 
The lowest value is presented by the aspect ratio of 1.0 and followed by 0.5 and 2.0 showing a low RMSE value of $0.086,0.227$ and 0.3089 , respectively.

Figure 9 is showing the post-processor of the S-version FEM, by exhibiting the stresses contour around the crack front. The higher stresses contour around the crack front is indicating that the crack will grow if the cyclic loading is applied continuously. The yellowish colour around the crack front indicates that the higher stress occurred, thus enabling the crack to propagate. Therefore, it supports the idea of the tendency for the crack to grow at a larger crack area besides having a higher SIF value. It is quite difficult to see the stresses variation for

Figure 9(a) and (c). However,

Figure 9(b) shows a clearer contour with brighter yellow colour indicating higher stresses occurring around the crack front. Figure 10 represents the comparison of the crack length versus the number of cycles with different aspect ratios. The results show a higher number of cycles recorded by a 1.0 aspect ratio compared with 0.5 and 2.0, correspondingly. The same behaviour was obtained by [40] where a lower fatigue life was predicted for the $\mathrm{a} / \mathrm{c} \neq 1.0$. The reason is due to a smaller size of the initial crack and steadier crack growth propagation. The 2.0 aspect ratio inclination is greater than the 1.0 and 0.5 aspect ratio. This is due to the higher SIF recorded by the 2.0 aspect ratio compared with 0.5 and 1.0, respectively. Therefore, the lowest number of cycles is recorded compared to the others.

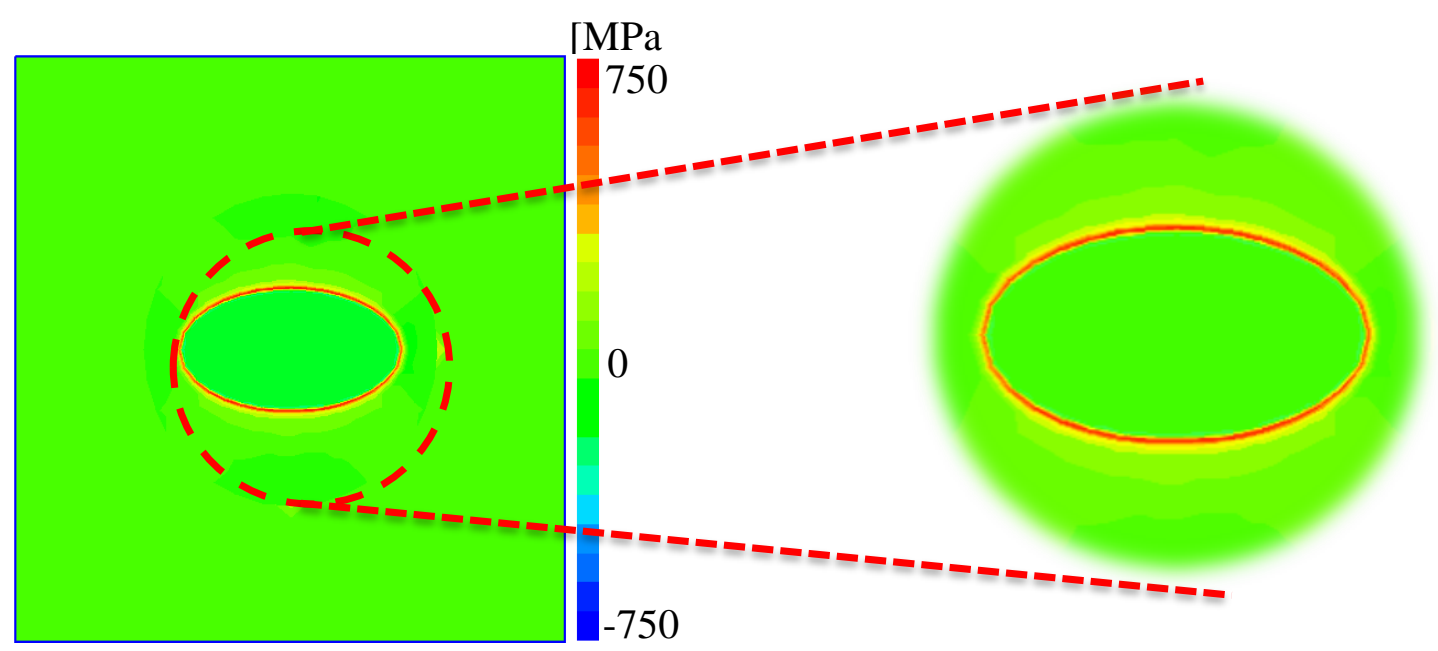

(a) 


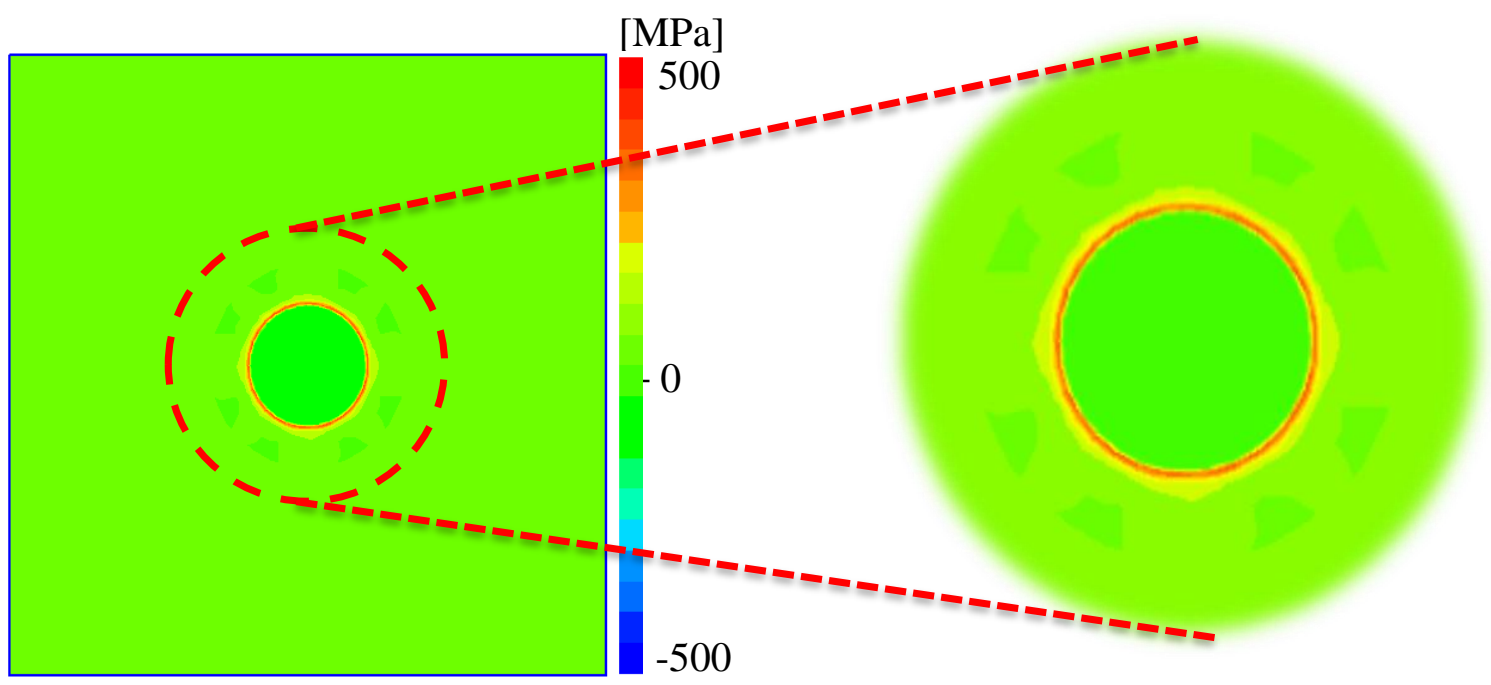

(b)

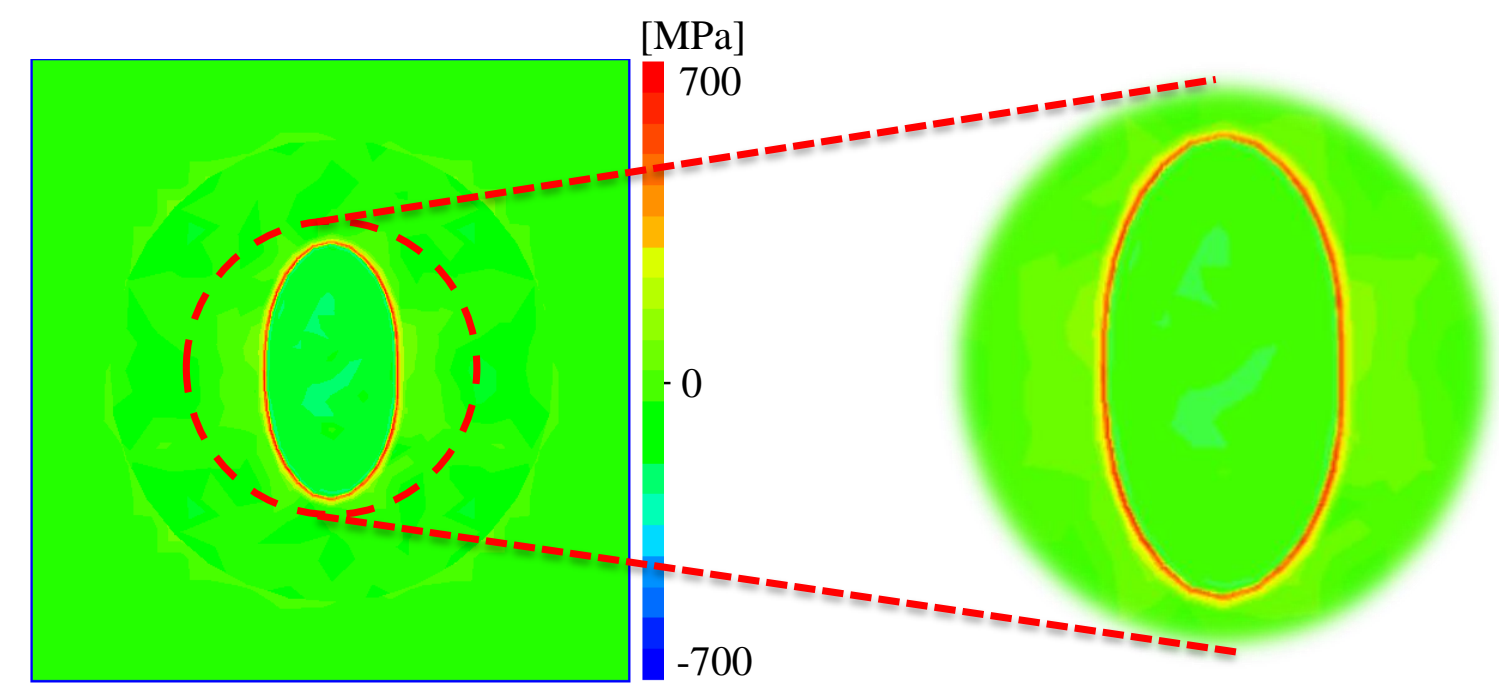

(c)

Figure 9. Behaviour of the stresses contour along the crack front corresponding to (a) 0.5 aspect ratio. (b) 1.0 aspect ratio. (c) 2.0 aspect ratio. 


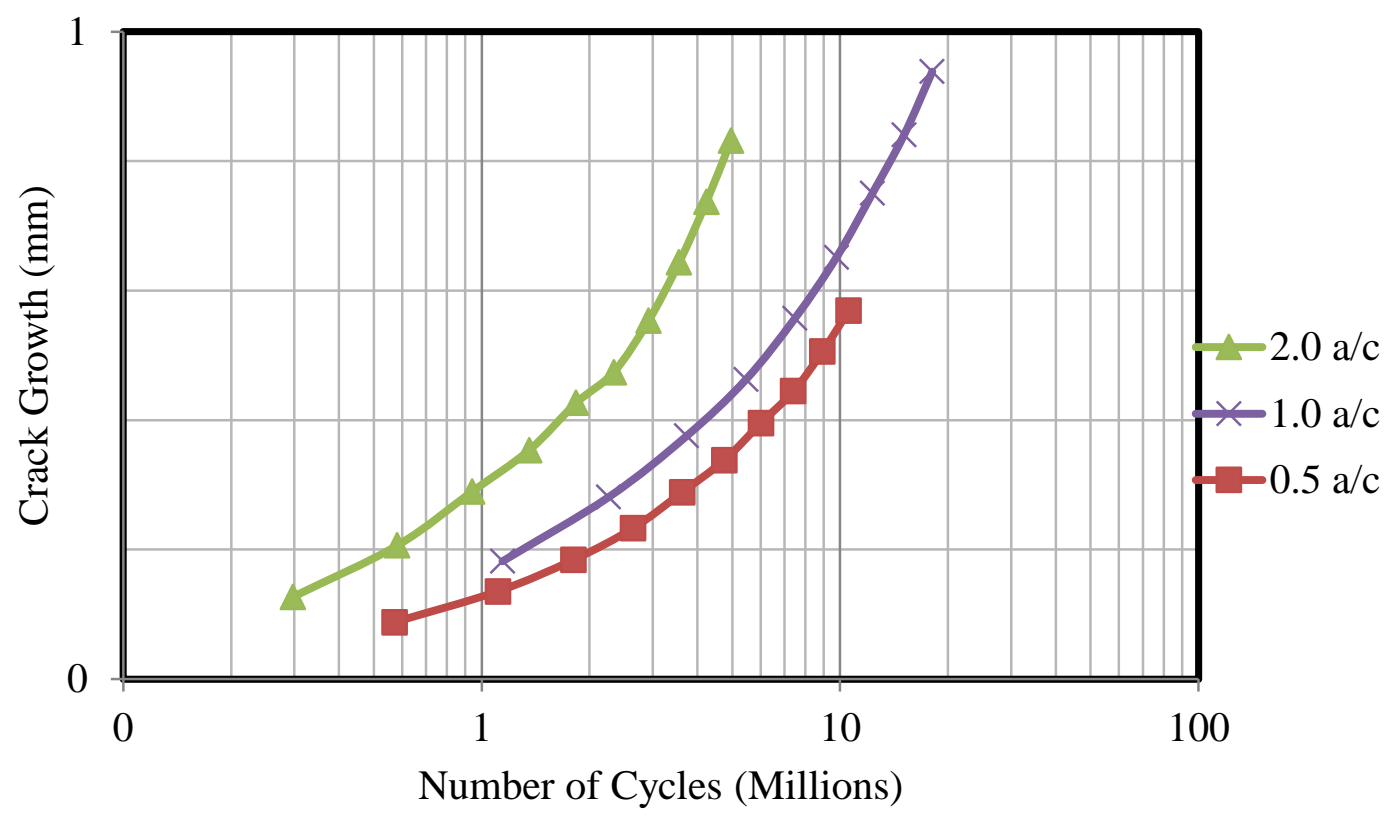

Figure 10. Comparison between crack growth and the number of cycles corresponding to the aspect ratios.

\section{CONCLUSIONS}

The computational S-version FEM integrated with VCCM is presented to predict the fatigue crack growth behaviour. This study adopted the superimposed FEM theory and used the automatically re-generated meshing. This will reduce the computational time and memory usage. The prediction of fatigue crack growth for an embedded crack using the S-version FEM was successfully performed under tension loading. Hence, the fatigue crack growth results were compared between different aspect ratios according to the SIF. The SIF value correlates with the tendency for the crack to grow. For a tight and sharp corner crack shape, a smaller value of SIF is obtained, hence preventing the crack to propagate. The wider areas are producing much higher SIF values compared with the tighter areas. Wherever the SIF value is large, it implicates the possibility for the crack to grow. Therefore, the highest value of SIF resulted in regions where the crack can easily propagate. Finally, the comparison between the S-version FEM and Newman \& Raju [30] shows a good agreement for the SIF corresponding to the aspect ratios. In addition, the correlation analysis was performed and a low RMSE value of $0.227,0.086$ and 0.3089 was obtained corresponding to the aspect ratio of $0.5,1.0$ and 2.0, respectively. Hence, proving the capability of the S-version FEM to simulate the fatigue embedded crack inside a prismatic bar.

\section{ACKNOWLEDGEMENTS}

The author would like to acknowledge the Universiti Kebangsaan Malaysia (UKM), especially the Laboratory of Computational \& Experimental Mechanics (CEM) for allowing the research to be conducted using the High Performance Computer (HPC). This research is supported by the Ministry of Higher Education under Fundamental Research Grant Scheme. FRGS/1/2015/TK03/UKM/01/2.

\section{REFERENCES}


[1] Mansor NII, Abdullah S, Ariffin AK, Syarif J. A review of the fatigue failure mechanism of metallic materials under a corroded environment. Engineering Failure Analysis. 2014;42:353-65.

[2] Qian G, Niffenegger M. Probabilistic fracture assessment of piping systems based on FITNET FFS procedure. Nuclear Engineering and Design. 2011;241:714-22.

[3] Kanto Y, Onizawa K, Machida H, Isobe Y, Yoshimura S. Recent Japanese research activities on probabilistic fracture mechanics for pressure vessel and piping of nuclear power plant. International Journal of Pressure Vessels and Piping. 2010;87:11-6.

[4] Hong SW, Koo JM, Seok CS, Kim JW, Kim JH, Hong SK. Fatigue life prediction for an API 5L X42 natural gas pipeline. Engineering Failure Analysis. 2015; 56:396-402.

[5] Mohamed SAN, Abdullah S, Arifin A, Ariffin AK, Padzi MM. Characterization of the biaxial fatigue behaviour on medium carbon steel using the strain-life approach. International Journal of Automotive and Mechanical Engineering. 2016;13:3262-77.

[6] Mahmud M, Abdullah S, Yunoh MFM, Ariffin AK, Nopiah ZM. Damaging fatigue cycles determination for random service loadings using mixed Weibull analysis. International Journal of Automotive and Mechanical Engineering. 2016;13:3628-41.

[7] Sivananth V, Vijayarangan S. Fatigue life analysis and optimization of a passenger car steering knuckle under operating conditions. International Journal of Automotive and Mechanical Engineering. 2015;11:2417-29.

[8] Lotsberg I, Sigurdsson G, Fjeldstad A, Moan T. Probabilistic methods for planning of inspection for fatigue cracks in offshore structures. Marine Structures. 2016;46:167-92.

[9] Shaari MS, Ariffin AK, Akiyuki T, Abdullah S, Kikuchi M, Akramin MRM. Prediction of fatigue crack growth for semi-elliptical surface cracks using Sversion fem under tension loading. Journal of Mechanical Engineering and Sciences. 2016;10:2375-86.

[10] Akramin MRM, Shaari MS, Ariffin AK, Kikuchi M, Abdullah S. Surface crack analysis under cyclic loads using probabilistic S-version finite element model. Journal of the Brazilian Society of Mechanical Sciences and Engineering. 2015;37:1851-65.

[11] Katsumata G, Li Y, Hasegawa K, Lacroix V. Remaining lives of fatigue crack growths for pipes with subsurface flaws and subsurface-to-surface flaw proximity rules. Journal of Pressure Vessel Technology. 2016;138:051402-.

[12] Ahmad MIM, Arifin A, Abdullah S. Evaluating effect of magnetic flux leakage signals on fatigue crack growth of mild steel. Journal of Mechanical Engineering and Sciences. 2016;10:1827-34.

[13] Ahmad MIM, Arifin A, Abdullah S. Evaluation of magnetic flux leakage signals on fatigue crack growth of mild steel. Journal of Mechanical Engineering and Sciences. 2015;9:1727-33.

[14] Kikuchi M, Wada Y, Suga K. Surface crack growth simulation under mixed mode cyclic loading condition. Procedia Engineering. 2011;10:427-32.

[15] Wu SY, Tsai BJ, Chen JJ. Elastic-plastic finite element analyses for reducers with constant-depth internal circumferential surface cracks. International Journal of Pressure Vessels and Piping. 2015;131:10-4. 
[16] Wu J-Y, Li F-B. An improved stable XFEM (Is-XFEM) with a novel enrichment function for the computational modeling of cohesive cracks. Computer Methods in Applied Mechanics and Engineering. 2015; 295: 77-107.

[17] Dündar H, Ayhan AO. Non-planar crack growth analyses of multiple cracks in thin-walled structures. International Journal of Fatigue. 2016; 96(2): 596-604.

[18] Sivananth V, S.Vijayarangan. Fatigue life analysis and optimization of a passenger car steering knuckle under operating conditions. International Journal of Automotive and Mechanical Engineering. 2015;11:2417-29.

[19] Kamal M, Rahman MM. Finite element-based fatigue behaviour of springs in automobile suspension. International Journal of Automotive and Mechanical Engineering. 2014;10:1910-9.

[20] Kamal M, Rahman MM. Fatigue life estimation based on continuum mechanics theory with application of genetic algorithm. International Journal of Automotive and Mechanical Engineering. 2015;11:2586-98.

[21] Almaraz GMD, Tapia MG, Silva EET, Calderón EC. Fatigue life prediction based on macroscopic plastic zone on fracture surface of AISI-SAE 1018 Steel. International Journal of Automotive and Mechanical Engineering. 2010;1:29-37.

[22] Hussain F, Abdullah S, Nuawi MZ. Effect of temperature on fatigue life behaviour of aluminium alloy AA6061 using analytical approach. Journal of Mechanical Engineering and Sciences. 2016;10:2324-35.

[23] Kamal M, Rahman MM, Rahman AGA. Fatigue life evaluation of suspension knuckle using multibody simulation technique. Journal of Mechanical Engineering and Sciences. 2012;3:291-300.

[24] Gu D, He B. Finite element simulation and experimental investigation of residual stresses in selective laser melted Ti-Ni shape memory alloy. Computational Materials Science. 2016;117:221-32.

[25] Zhang YM, Fan M, Xiao ZM, Zhang WG. Fatigue analysis on offshore pipelines with embedded cracks. Ocean Engineering. 2016;117:45-56.

[26] Patelli E, Murat Panayirci H, Broggi M, Goller B, Beaurepaire P, Pradlwarter HJ, et al. General purpose software for efficient uncertainty management of large finite element models. Finite Elements in Analysis and Design. 2012;51:31-48.

[27] Fish J. The s-version of the finite element method. Computers \& Structures. 1992;43:539-47.

[28] Okada H, Kawai H, Tokuda T, Fukui Y. Fully automated mixed mode crack propagation analyses based on tetrahedral finite element and VCCM (virtual crack closure-integral method). International Journal of Fatigue. 2013;50:33-9.

[29] Wada Y, Kikuchi M, Yamada S, Serizawa R, Li Y. Fatigue growth of internal flaw: Simulation of subsurface crack penetration to the surface of the structure. Engineering Fracture Mechanics. 2014;123:100-15.

[30] Newman Jr JC, Raju IS. Stress-intensity factor equations for cracks in threedimensional finite bodies subjected to tension and bending loads. NASA Technical Memorandum 85793. 1984.

[31] Kikuchi M, Wada Y, Li Y. Crack growth simulation in heterogeneous material by S-FEM and comparison with experiments. Engineering Fracture Mechanics. 2016.

[32] Rybicki EF, Kanninen MF. A finite element calculation of stress intensity factors by a modified crack closure integral. Engineering Fracture Mechanics. 1977;9:931-8. 
[33] Paris P, Erdogan F. A Critical Analysis of Crack Propagation Laws. Journal of Basic Engineering. 1963;85:528-33.

[34] Richard HA, Sander M, Fulland M, Kullmer G. Development of fatigue crack growth in real structures. Engineering Fracture Mechanics. 2008;75:331-40.

[35] Richard HA, Fulland M, Sander M. Theoretical crack path prediction. Fatigue \& Fracture of Engineering Materials \& Structures. 2005;28:3-12.

[36] Miyamoto H, Kikuchi M, Okazaki T, Kubo M. First Special Issue on SMiRT7The J integral evaluation of a nozzle corner crack under thermal transient loading condition. Nuclear Engineering and Design. 1983;75:213-22.

[37] Okada H, Higashi M, Kikuchi M, Fukui Y, Kumazawa N. Three dimensional virtual crack closure-integral method (VCCM) with skewed and non-symmetric mesh arrangement at the crack front. Engineering Fracture Mechanics. 2005;72:1717-37.

[38] Gardin C, Fiordalisi S, Sarrazin-Baudoux C, Gueguen M, Petit J. Numerical prediction of crack front shape during fatigue propagation considering plasticityinduced crack closure. International Journal of Fatigue. 2016;88:68-77.

[39] Jin HJ, Wu SJ. A new driving force parameter for fatigue growth of multiple cracks. International Journal of Fatigue. 2017;96:10-6.

[40] Chandra D, Purbolaksono J, Nukman Y, Liew HL, Ramesh S, Hamdi M. Fatigue crack growth of a corner crack in a square prismatic bar under combined cyclic torsion-tension loading. International Journal of Fatigue. 2014;64:67-73. 